\title{
US officials warn 39 countries about risk of hantavirus among travellers to Yosemite
}

In this News story by Bob Roehr (BMJ 2012;345:e6054, doi:10. 1136/bmj.e6054), we mistakenly referred to Vanya Gant, the London infectious disease specialist mentioned in the last two paragraphs, as "she" and not he as is correct. We would like to apologise to Vanya Gant for this mistake.
Cite this as: BMJ 2012;345:e6295

๑) BMJ Publishing Group Ltd 2012 